\title{
Pretreatment prognostic nutritional index is a prognostic marker for extensive-stage small cell lung cancer patients treated with anlotinib
}

\author{
Jingjing Liu ${ }^{1}$, Shuang $\mathrm{Li}^{2}$, Shuang Zhang ${ }^{2}$, Changliang Yang ${ }^{2}$, Liang Zhang ${ }^{2}$, Bin Zhang ${ }^{1}$, Ying Cheng ${ }^{2}$, \\ Changli Wang ${ }^{1}$
}

${ }^{1}$ Department of Lung Cancer, Tianjin Medical University Cancer Institute and Hospital, National Clinical Research Center for Cancer, Key Laboratory of Cancer Prevention and Therapy, Tianjin's Clinical Research Center for Cancer, Tianjin Lung Cancer Center, Tianjin, China; ${ }^{2}$ Department of Thoracic Oncology, Jilin Provincial Cancer Hospital, Changchun, China

Contributions: (I) Conception and design: C Wang, Y Cheng; (II) Administrative support: C Wang, Y Cheng; (III) Provision of study materials or patients: J Liu, S Zhang, C Yang, L Zhang; (IV) Collection and assembly of data: J Liu, S Li; (V) Data analysis and interpretation: S Li, B Zhang; (VI) Manuscript writing: All authors; (VII) Final approval of manuscript: All authors.

Correspondence to: Changli Wang. Department of Lung Cancer, Tianjin Lung Cancer Center, Tianjin Medical University Cancer Institute and Hospital, Huan-Hu-Xi Road, Ti-Yuan-Bei, He Xi District, Tianjin 300060, China. Email: wangchangli309@163.com; Ying Cheng, Department of Thoracic Oncology, Jilin Provincial Cancer Hospital, No.1018 Hu Guang Road, Changchun 130012, China. Email: chengying@csco.org.cn.

Background: Anlotinib is a multi-targeted tyrosine kinase inhibitor that inhibits tumor angiogenesis which has shown activity in several malignancies and approved for the treatment of small cell lung cancer (SCLC) in China. However, there are no markers can predict the clinical outcomes of anlotinib. We aimed to evaluate the efficacy of anlotinib in extensive stage SCLC (ES-SCLC) patients who failed at least two regimens treatment and to explore potential factors related to its survival benefit.

Methods: Patients with ES-SCLC treated with anlotinib monotherapy were screened between March 2017 and May 2019, prognostic nutritional index (PNI) before treatment were collected. Progression free survival (PFS) and overall survival (OS) were calculated and compared using the Kaplan-Meier method and the log-rank test. The prognostic values of each variable were evaluated with univariate and multivariate Cox proportional hazard regression (PHR) analyses.

Results: A total of 41 patients with ES-SCLC were received anlotinib treatment, the median age was 57 (range, 33-76). Median OS was significantly longer in the PNI high arm compared with the low arm [8.4 months (95\% CI, 5.1-9.6 months) vs. 4.7 months (95\% CI, 2.1-6.3 months); hazard ratio (HR) 0.42 (95\% CI, 0.21-0.85); P=0.01]. The median PFS of two arms were 4.1 months (95\% CI, 2.1-8.2 months) and 2.6 months (95\% CI, 0.7-3.9 months), respectively ( $\mathrm{HR}=0.53,95 \% \mathrm{CI}, 0.27-1.02, \mathrm{P}=0.05$ ). Multivariate analysis confirmed that $\mathrm{PNI}(\mathrm{P}<0.01)$ and $\mathrm{LDH}(\mathrm{P}<0.01)$ were significant independent biomarkers for OS.

Conclusions: The present study demonstrated that pretreatment PNI can be used as a novel and convenient biomarker to predict the prognosis in ES-SCLC patients treated with anlotinib.

Keywords: Small cell lung cancer (SCLC); prognostic nutritional index (PNI); anlotinib; prognostic factor

Submitted Feb 05, 2020. Accepted for publication Aug 21, 2020.

doi: $10.21037 /$ jtd-20-755

View this article at: http://dx.doi.org/10.21037/jtd-20-755

(C) Journal of Thoracic Disease. All rights reserved. 


\section{Introduction}

Small-cell lung cancer accounts for $14 \%$ of lung cancers (1), and is the most lethal subtype with rapid doubling time, high growth fraction and early metastases (2). Most patients are diagnosed as extensive-stage, and the prognosis is quite poor for a survival only $2-4$ months without treatment (3). The rapid development of precision medicine leads to revolutionary changes in management of non-small cell lung cancer (NSCLC), while the treatment of SCLC remains relatively stagnant especially for third-line and further-line treatment. Although checkpoint inhibitors such as nivolumab and pembrolizumab were successively approved by Food and Drug Administration (FDA) for third-line and further-line treatment $(4,5)$, but these two drugs have not approved in China for SCLC indication.

Anlotinib is an innovative multi-target tyrosine kinase inhibitor that inhibit vascular endothelial growth factor receptor (VEGFR)1/2/3, fibroblast growth factor receptor (FGFR)1-4, platelet-derived growth factor receptor (PDGFR) $\alpha / \beta$, stem cell factor receptor (c-Kit) (6). In China, anlotinib is approved for third-line therapy for advanced NSCLC and second-line treatment for soft tissue sarcoma. For SCLC, based on ALTER1202 study, a phase 2 study demonstrated that anlotinib as third-line or subsequent treatment for SCLC showed longer progression-free survival (PFS) and overall survival (OS) than placebo with favorable safety profile, anlotinib was approved by National Medical Products Administration (NMPA) for third-line treatment of SCLC in August 2019. Although anlotinib became the first targeted drug to provide survival benefits to SCLC, but the overall response rates of anlotinib are modest and still lack of the ideal predictive biomarkers for the efficacy of anti-angiogenic therapy and many heavytreated SCLC patients could not provide sufficient tumor tissue for testing. Explore markers to predict the clinical outcomes of anlotinib have high unmet needs.

Studies have shown that systemic inflammation is related to tumor proliferation, angiogenesis and metastasis $(7,8)$. The responsive of systemic inflammatory has a definite effect on progressive nutrition, dysfunction and poor prognosis in tumor patients $(8,9)$. Poor nutritional status was closely associated with poor outcome in a various of cancers $(10,11)$. Onodera et al. proposed the concept of prognostic nutritional index (PNI) which is the mostly used marker of nutritional status (12). PNI is based on serum albumin concentrations and total lymphocyte counts in the peripheral blood and evaluates the potential impact of hypoalbuminemia and lymphocytopenia. Lymphocytes are an important cellular component of the inflammatory and immune systems, and also an indicator of poor prognosis. Hypoalbuminemia is not only an indicator of nutrient deficiency, but also suggests an increase in systemic inflammatory status. PNI was originally used to assess preoperative risk of colorectal cancer and as a determinant of surgical indications. Subsequently, PNI is widely used to evaluate the nutritional status of tumor patients. Several studies have suggested that PNI is a useful prognostic factor after treatment in patients with gastric cancer, colorectal cancer, liver cancer and lung cancer (13-17). For SCLC, researches have confirmed that pretreatment PNI can be a better predictor for the prognosis of SCLC and low pretreatment PNI is correlated with poor OS or PFS of SCLC patients (18-20). PNI can also indicate systemic inflammation which affects the growth and survival of tumor cells, promotes angiogenesis, metastasis and decreasing tumor sensitivity to treatments (21). Low pretreatment PNI can predict poor outcomes in patients with metastatic renal cell carcinoma or hepatocellular carcinoma treated by sunitinib or sorafenib (22-24).

However, the association between anlotinib and PNI has not been reported. Therefore, we conducted this retrospective study to explore the correlation between pretreatment PNI and survival in patients with extensive stage SCLC (ES-SCLC) treated with anlotinib. We present the following article in accordance with the REMARK reporting checklist (available at http://dx.doi.org/10.21037/ jtd-20-755).

\section{Methods}

\section{Patient enroll criteria and therapeutic procedure}

This study included patients with ES-SCLC treated with anlotinib for third-line or further-line treatment at the Jilin Provincial Cancer Hospital between March 2017 and May 2019 were enrolled in this study. The inclusion criteria were as follows: (I) cytologically or histologically confirmed ES-SCLC, (II) patients have progressed from at least 2 lines of chemotherapy, (III) available laboratory data from pretreatment blood samples, (IV) haven't received antitumor therapy within 2 weeks before blood draw, including cytotoxic therapy, radiotherapy, other experimental anticancer therapy, (V) blood must be collected and tested within 7 days prior to anlotinib treatment, (VI) standard of blood routine test (no blood transfusion within 14 days), 
hemoglobin $(\mathrm{HB}) \geq 90 \mathrm{~g} / \mathrm{L}$, absolute value of neutrophils $(\mathrm{ANC}) \geq 1.5 \times 10^{9} / \mathrm{L}$, platelets $(\mathrm{PLT}) \geq 80 \times 10^{9} / \mathrm{L}$, (VII) with sufficient medical information. All patients received anlotinib in a standard dose regimen, i.e., $12 \mathrm{mg} /$ day, in $2 / 1$ week cycles. Data were collected from the medical records including: demographic data, treatment information, best response classified according to Response Evaluation Criteria In Solid Tumors (RECIST) 1.1 criteria, results of hematologic tests before anlotinib treatment. The data was analyzed by professional statisticians, treatment records were evaluated by clinical experienced doctors. The last follow-up was on August 20, 2019.

The study was conducted in accordance with the Declaration of Helsinki (as revised in 2013) and the Harmonized Tripartite Guideline for Good Clinical Practice from the International Conference on Harmonization. This study was approved by Jilin Provincial Cancer Hospital ethic committee. In addition, all patients have signed the informed consent before receiving the anlotinib treatment.

\section{Assessment of response and calculation of the pretreatment PNI}

Responses to treatment were evaluated as complete response (CR), partial response (PR), stable disease (SD), and progressive disease (PD) according to the RECIST version 1.1. A third higher level physician examined the information above. All disagreements were resolved by discussion between three doctors until the consensus was reached. All patients received routine hematologic tests within seven days before the administration of anlotinib. The PNI was calculated as albumin $(\mathrm{g} / \mathrm{L})+5 \times$ lymphocyte $\left(\times 10^{9} / \mathrm{L}\right)$ and patients with an PNI $<45$ was considered as abnormal, as described previously (25). Body mass index (BMI) was calculated as body weight divided by height squared $\left(\mathrm{kg} / \mathrm{m}^{2}\right)$, and classified into 3 categories: underweight $\left(\mathrm{BMI}<18.5 \mathrm{~kg} / \mathrm{m}^{2}\right)$, normal $\left(18.5 \mathrm{~kg} / \mathrm{m}^{2} \leq \mathrm{BMI}\right.$ $<24.0 \mathrm{~kg} / \mathrm{m}^{2}$ ), while overweight or obese was defined if the $\mathrm{BMI} \geq 24.0 \mathrm{~kg} / \mathrm{m}^{2}$.

\section{Statistical analyses}

OS was measured from treatment initiation until death. Patients who were still alive were censored at the final follow-up. PFS was defined as the interval from treatment initiation until disease progression or death. Patients still manifested disease control were censored at the final follow-up. The overall response rate (ORR) included the
$\mathrm{CR}$ and PR. Disease control rate (DCR) was defined as the proportion of patients whose CR or PR or SD lasted longer than 24 weeks.

Descriptive analysis was used for all variables. Counting variables were presented as percentages. The area under the curve (AUC) of receiver operating characteristics (ROC) curve was calculated to evaluate the discriminatory ability. PFS and OS were calculated and compared using the Kaplan-Meier method and the log-rank test. Multivariate Cox proportional hazards models was used to evaluate whether PNI or other baseline characteristics were associated with OS or PFS, include age, sex, BMI, ECOG PS, smoking history, CNS metastasis, liver metastasis, LDH and PNI. To arrive at the final multivariate model, all candidate variables were included whether which were significant on univariate analysis or not with enter selection. $\mathrm{P}<0.05$ was considered statistically significant. Hazard ratios (HRs) and 95\% CIs were calculated. All analyses were statistically analyzed using SPSS24.0 (Chicago, Illinois, USA) and R 3.4.3.

\section{Results}

\section{Patients}

The characteristics of patients are summarized in Table 1. A total of 41 patients with ES-SCLC treated with oral anlotinib (12 mg/d) were enrolled in this study. Each cycle was defined as 2 weeks on-treatment followed by 1 week off-treatment. The treatment continued until disease progression or treatment intolerance. Among the 41 patients, the median age at diagnosis was 57 (range, $33-76)$ years and 31 (75.6\%) were male. CNS metastases were present at baseline in 18 (43.9\%) patients. According to the cutoff value of 45,25 patients $(61.0 \%)$ divided into PNI high arm (PNI $\geq 45)$ and 16 patients $(39.0 \%)$ in PNI low arm (PNI <45). For OS, the AUC of ROC was 0.689 (sensitivity $=90 \%$; specificity $=51.6 \%$ ); for $\mathrm{PFS}$, the AUC of ROC was 0.730 (sensitivity $=97.3 \%$; specificity $=50 \%)$. The demographic and disease characteristics were generally well balanced between the two arms.

\section{Efficacy of anlotinib in overall patients}

At the time of data cutoff, the median PFS was 3.5 months (95\% CI, 2.0-4.5) (Figure 1A). The median OS was 6.4 months (95\% CI, 4.6-8.4) (Figure 1B) and all patients died due to progression and tumor-related complications. 
Table 1 Characteristics of patients

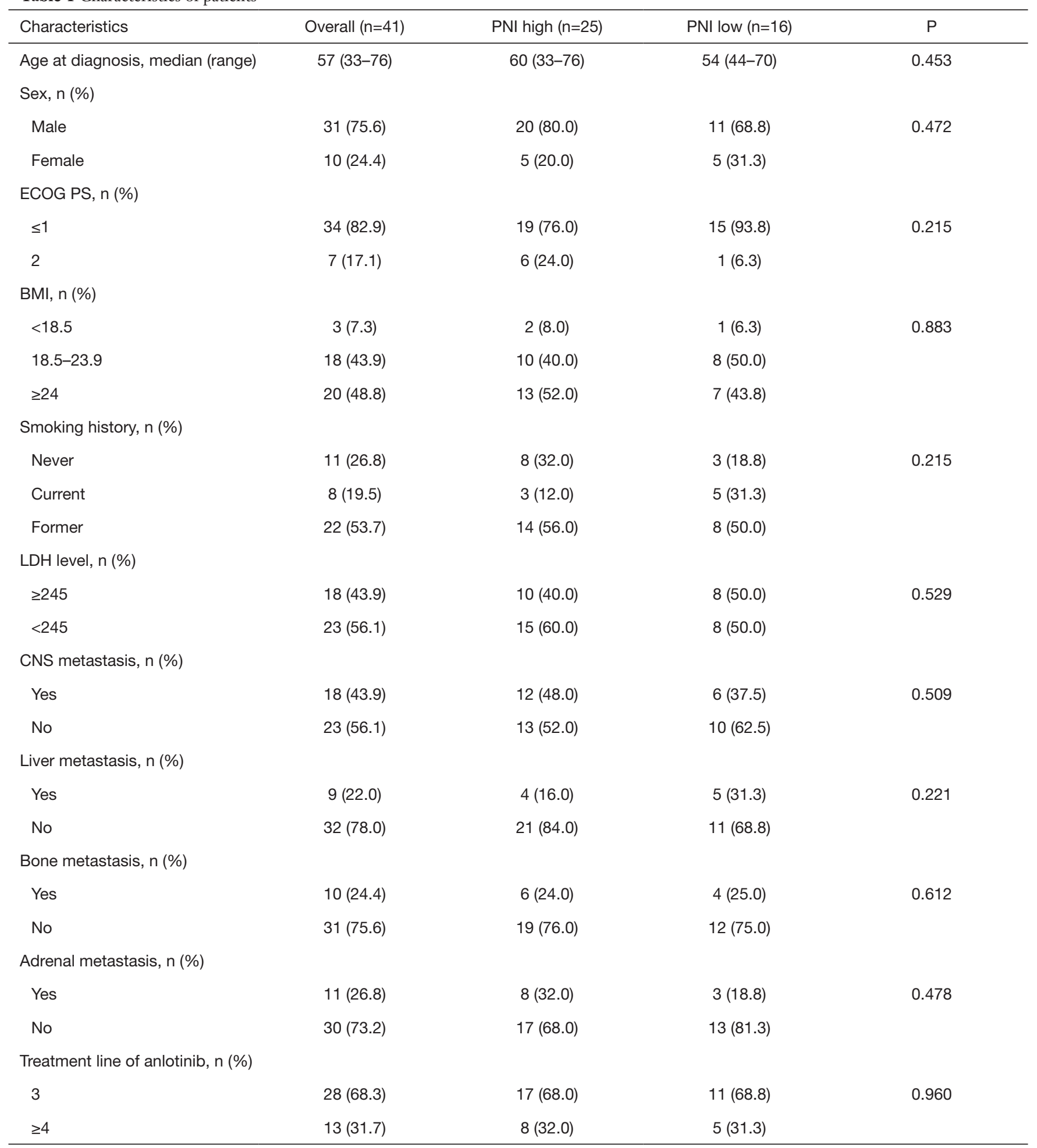

ECOG PS, Eastern Cooperative Oncology Group performance status; BMI, body mass index; LDH, lactate dehydrogenase; CNS, central nervous system. 

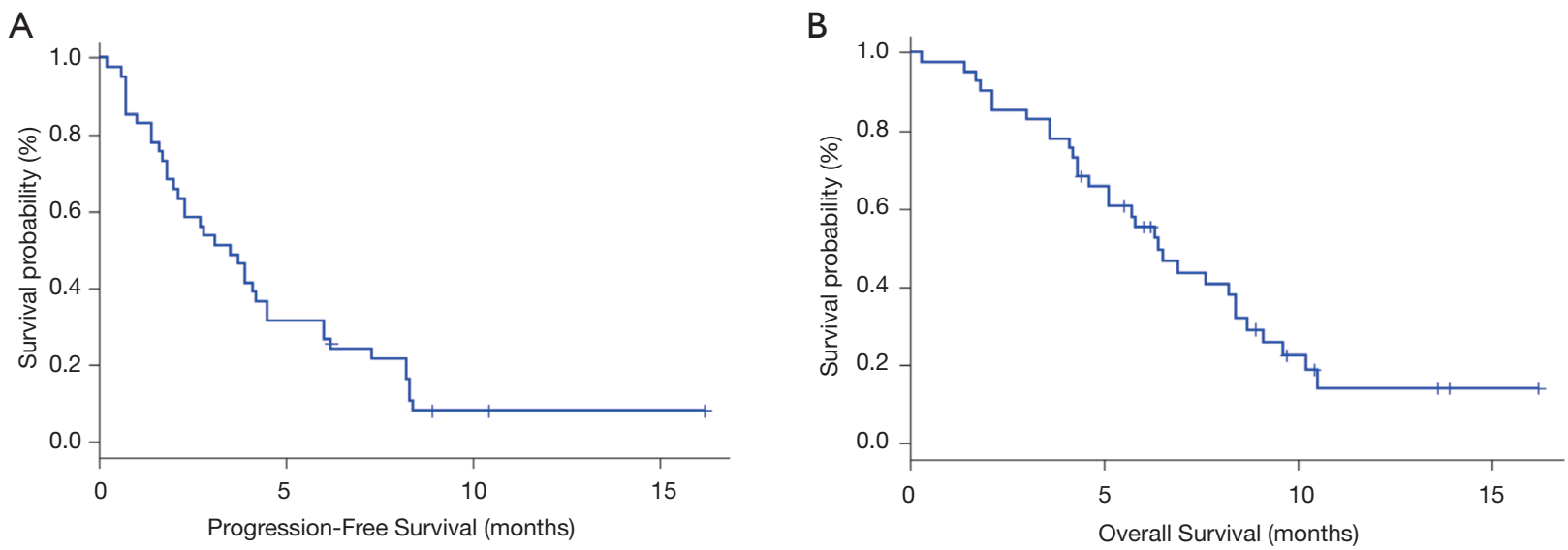

Figure 1 Kaplan-Meier curves for progression-free survival (A) and overall survival (B) for all patients.

Table 2 Tumor response

\begin{tabular}{|c|c|c|c|c|}
\hline$N(\%)$ & Overall $(n=41)$ & PNI high $(n=25)$ & PNI low (n=16) & $\mathrm{P}$ value (PNI high vs. PNI low) \\
\hline CR & 0 & 0 & 0 & - \\
\hline PR & $2(4.9 \%)$ & $2(8.0 \%)$ & 0 & - \\
\hline SD & $32(78.0 \%)$ & $20(80.0 \%)$ & $12(75.0 \%)$ & - \\
\hline DCR & $34 / 41(82.9 \%)$ & $22 / 25$ (88.0\%) & $12 / 16(75.0 \%)$ & $<0.001$ \\
\hline
\end{tabular}

ORR, overall response rate; CR, complete response; PR, partial response; SD, stable disease; PD, progressive disease; DCR, disease control rate.

ORR was $4.9 \%$ (2/41), DCR was $82.9 \%(34 / 41)$ (Table 2). No patient achieved complete response, $4.9 \%$ achieved partial response, $78.0 \%$ had stable disease, and $17.1 \%$ had progressive disease.

\section{Relationship between pretreatment PNI and survival}

The median OS was longer in the PNI high arm (8.4 months; 95\% CI, 5.1-9.6 months) compared with the PNI low arm (4.7 months, 95\% CI, 2.1-6.3 months), HR 0.42; 95\% CI, 0.21-0.85; $\mathrm{P}=0.01$ (Figure $2 A$ ). The median PFS was 4.1 months (95\% CI, 2.1-8.2 months) for the PNI high arm, which was numerically longer than the median PFS for the PNI low arm 2.6 months (95\% CI, 0.7-3.9 months), but with no statistic difference (HR 0.53; 95\% CI, 0.27-1.02; $\mathrm{P}=0.05$ ) (Figure 2B). The ORR, assessed according to RECIST 1.1 , were $8.0 \%$ in PNI high arm and $0 \%$ in PNI low arm $(\mathrm{P}<0.001)$, respectively. DCR also improved (88.0\% vs. $75.0 \%, \mathrm{P}<0.001)$ (Table 2).

\section{Prognostic factors in ES-SCLC patients treated with anlotinib therapy}

We performed univariate and multivariate analyses of OS and PFS using COX regression model, and factors considered included age, sex, ECOG PS, smoking status, BMI, CNS Metastasis, liver metastasis, pretreatment PNI and LDH level. Univariate analysis showed that PNI (HR 2.39; 95\% CI, 1.17-4.88; P=0.01), LDH (HR 3.93; 95\% CI $(1.80-8.59 ; \mathrm{P}<0.01)$ were significantly associated with OS; liver metastasis (HR 2.41; 95\% CI, 1.12-5.18; $\mathrm{P}=0.03$ ), and LDH (HR 1.99; 95\% CI, 1.03-3.86; $\mathrm{P}=0.04$ ) were significantly affected PFS. Pretreatment PNI showed a trend towards being a factor for PFS but did not reach the level of statistical significance (HR 1.90; 95\% CI, 0.98-3.68, $\mathrm{P}=0.05$ ).

In multivariate analysis, the results demonstrated that PNI (HR 3.90; 95\% CI, 1.68-9.06), $\mathrm{P}<0.01)$ and LDH (HR 4.95; 95\% CI, 1.88-13.05; $\mathrm{P}<0.01)$ were independent prognostic 

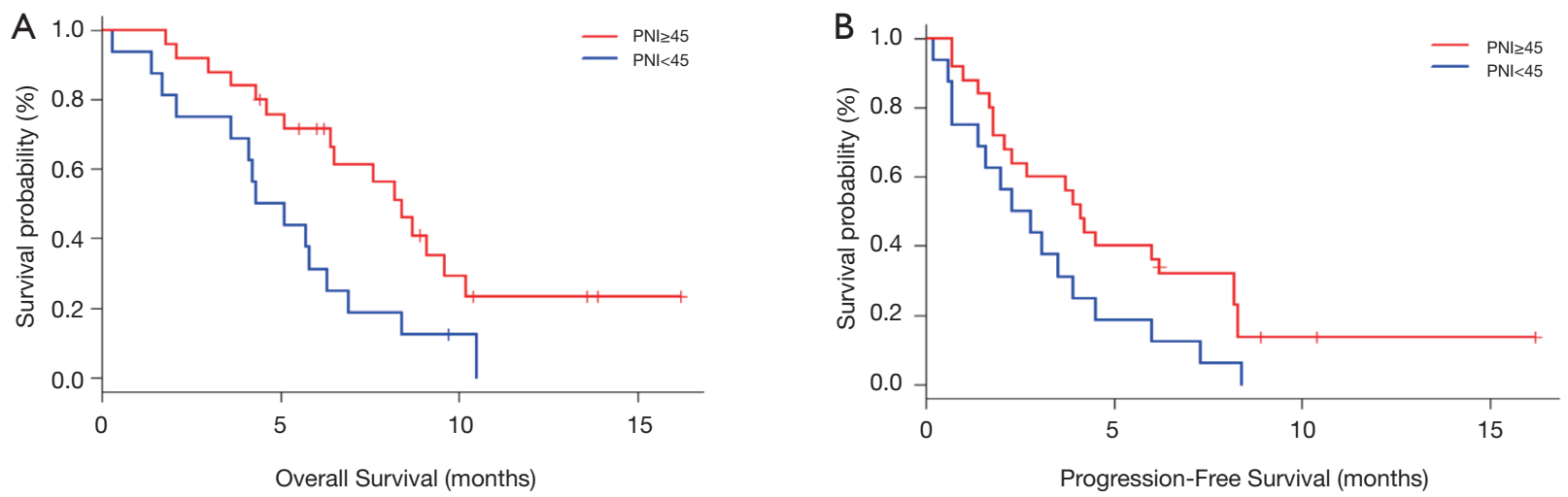

Figure 2 Kaplan Meier survival curves for overall survival (A) and progression-free survival (B) according to PNI level in ES-SCLC patients. PNI, prognostic nutritional index; ES-SCLC, extensive stage small cell lung cancer.

Table 3 Univariate and multivariate analysis of OS

\begin{tabular}{|c|c|c|c|c|}
\hline Characteristics & \multicolumn{2}{|c|}{ Univariate } & \multicolumn{2}{|c|}{ Multivariate } \\
\hline Age at diagnosis & $1.00(0.95-1.03)$ & 0.53 & $1.00(0.96-1.05)$ & 0.98 \\
\hline Sex (male vs. female) & $1.44(0.64-3.25)$ & 0.38 & $2.30(0.75-7.04)$ & 0.15 \\
\hline $\mathrm{BMI}$ & $0.97(0.88-1.07)$ & 0.49 & $0.97(0.85-1.11)$ & 0.67 \\
\hline Smoking history (ever vs. never) & $1.13(0.53-2.41)$ & 0.75 & $0.95(0.35-2.58)$ & 0.92 \\
\hline CNS metastasis (yes vs. no) & $0.97(0.47-2.00)$ & 0.93 & $0.81(0.29-2.23)$ & 0.68 \\
\hline Liver metastasis (yes vs. no) & $1.42(0.61-3.32)$ & 0.41 & $1.08(0.37-3.16)$ & 0.89 \\
\hline LDH ( $\geq 245$ vs. <245) & $3.93(1.80-8.59)$ & $<0.01$ & $4.95(1.88-13.05)$ & $<0.01$ \\
\hline
\end{tabular}

ECOG PS, Eastern Cooperative Oncology Group performance status; BMI, body mass index; LDH, lactate dehydrogenase; CNS, central nervous system.

factors for OS. PFS was significant affected by pretreatment sex (HR 3.54; 95\% CI, 1.16-10.76; P=0.03) and PNI (HR $3.55 ; 95 \% \mathrm{CI}, 1.52-8.33 ; \mathrm{P}<0.01)$. All of univariate and multivariate survival analyses are presented in Tables 3,4 .

\section{Discussion}

Anlotinib was the first targeted drug approved for third-line and further-line treatment of SCLC. In this study, we focused on the predictive value of nutritional status in the outcomes of ES-SCLC. The results suggested that pretreatment PNI was significantly related to ORR and DCR in ES-SCLC patients treated with anlotinib. Furthermore, PNI was found to be a prognostic factor for OS.
There is increasing evidence that inflammation is an important factor in the development and progression of cancer (26). In addition, the products of inflammatory processes are considered to be potential biomarkers of tumor microenvironment because leukocyte infiltration is interconnected with angiogenesis and VEGF related molecules are contribute to the recruitment of monocytes into primary tumors (7). As a wildly used marker of nutritional statues, PNI is a particularly biomarker because it calculated from albumin and lymphocyte count. PNI can reflects the inflammatory system as well as the nutritional status. On the one hand, as one of the key immune cell, lymphocyte can indicate lymphocyte-mediated immune response to tumors (7). On the other hand, serum albumin 
Table 4 Univariate and multivariate analysis of PFS

\begin{tabular}{|c|c|c|c|c|}
\hline Characteristics & \multicolumn{2}{|c|}{ Univariate } & \multicolumn{2}{|c|}{ Multivariate } \\
\hline Age at diagnosis & $1.01(0.97-1.04)$ & 0.83 & $1.00(0.97-1.05)$ & 0.77 \\
\hline Sex (male vs. female) & $1.69(0.79-3.62)$ & 0.17 & $3.54(1.16-10.76)$ & 0.03 \\
\hline BMI & $0.99(0.90-1.08)$ & 0.77 & $0.94(0.84-1.06)$ & 0.33 \\
\hline Smoking history (ever vs. never) & $1.17(0.58-2.37)$ & 0.67 & $0.68(0.28-1.65)$ & 0.40 \\
\hline CNS metastasis (yes vs. no) & $0.87(0.45-1.66)$ & 0.66 & $0.92(0.40-2.14)$ & 0.85 \\
\hline Liver metastasis (yes vs. no) & $2.41(1.12-5.18)$ & 0.03 & $1.94(0.76-4.91)$ & 0.16 \\
\hline LDH ( $\geq 245$ vs. <245) & $1.99(1.03-3.86)$ & 0.04 & $2.10(1.00-4.41)$ & 0.05 \\
\hline
\end{tabular}

ECOG PS, Eastern Cooperative Oncology Group performance status; BMI, body mass index; LDH, lactate dehydrogenase; CNS, central nervous system.

is known as a meaningful indicator that reflects both the inflammatory reaction and nutritional status and has been shown to be independently associated with prognosis of patients with many cancers (27-29). In some studies, PNI has been verified play an important prognostic role in different types of cancers such as lung cancer, gastric cancer, pancreatic cancer and oral squamous cell carcinoma (11,30-32). Lung cancer patients with lower PNI is associated with adverse tumor features, such as large size, poor differentiation and metastasis (21). In SCLC patients treated with platinum-based chemotherapy, pretreatment PNI was a good biomarker for the assessment of SCLC prognosis (20).

As the only approved targeted drug for the treatment of SCLC, anlotinib has been widely used in the clinical treatment of SCLC, but the value of PNI in the prognosis and efficacy of anlotinib is still inconclusive. Other antiangiogenic drugs such as bevacizumab for ovarian cancer and sorafenib for metastatic renal cell cancer have also observed a correlation between PNI and the clinical outcomes of anti-angiogenic drugs (24,33). Consistent with these observations, in the present study, our data confirmed that in patients with ES-SCLC, high pretreatment PNI had longer OS of anlotinib treatment. Although there was no significant difference in PFS between PNI high and PNI low arm, the value was numerically longer for PNI high patients. The efficacy of anlotinib was also favorable in PNI high arm, both ORR and DCR have obvious advantages.

Univariate and multivariate analysis was performed to assess the potential prognostic value of clinical characteristics and PNI level. The results further confirmed that high PNI before treatment was an independent prognostic factor for OS in patients with ES-SCLC treated with anlotinib, and PNI was also an independent prognostic factor for PFS in the multivariate analysis. In addition, the results of univariate analysis also found that LDH level was an independent prognostic factor for both OS and PFS. However, in the multivariate analysis, $\mathrm{LDH}$ level was only an independent prognostic factor for OS, suggesting that $\mathrm{LDH}$ level is more valuable in evaluating OS of anlotinib. We have also investigated the impact of pretreatment BMI on the prognosis of anlotinib. But regardless of PFS or OS, the multivariate analysis suggested that BMI had no significant correlation, prompt BMI may be not an independent prognostic factor of anlotinib. It is important to note that although we observed a correlation between PNI and prognosis in ES-SCLC patients, PNI has not been used in clinical practice and is well worth further study to confirm.

This finding is the first observation to show the relationship between the PNI and anlotinib efficacy in cancer patients. PNI is a readily obtained and is more acceptable to patients with higher effectiveness-cost ratio. However, as a retrospective and single center study, there are some limitations including small cohort size, lack of a validation group and potential missing data, which may need further verified by prospective study. Finally, because anlotinib is only approved in China at present, the population of this study is only Chinese patients, so relevant exploration study 
of other races needs to be carried out in the future.

\section{Conclusions}

The present study suggested that pretreatment high PNI is an independent better prognostic predictor in ES-SCLC patients received anlotinib for third-line or further-line treatment. Further studies are needed to demonstrate this advantage.

\section{Acknowledgments}

Funding: This work was supported by grants from the Training program for Scientific and technological backbone of Jilin Province health and wellness (No. 2018Q006).

\section{Footnote}

Reporting Checklist: The authors have completed the REMARK reporting checklist. Available at http://dx.doi. org/10.21037/jtd-20-755

Data Sharing Statement: Available at http://dx.doi. org/10.21037/jtd-20-755

Peer Review File: Available at http://dx.doi.org/10.21037/jtd20-755

Conflicts of Interest: All authors have completed the ICMJE uniform disclosure form (available at http://dx.doi. org/10.21037/jtd-20-755). The authors have no conflicts of interest to declare.

Ethical Statement: The authors are accountable for all aspects of the work in ensuring that questions related to the accuracy or integrity of any part of the work are appropriately investigated and resolved. The study was conducted in accordance with the Declaration of Helsinki (as revised in 2013) and the Harmonized Tripartite Guideline for Good Clinical Practice from the International Conference on Harmonization. The study was reviewed and approved by ethics committee of Jilin Provincial Cancer Hospital (approval number 201701-002-01). All patients enrolled completed the informed consent form.

Open Access Statement: This is an Open Access article distributed in accordance with the Creative Commons Attribution-NonCommercial-NoDerivs 4.0 International
License (CC BY-NC-ND 4.0), which permits the noncommercial replication and distribution of the article with the strict proviso that no changes or edits are made and the original work is properly cited (including links to both the formal publication through the relevant DOI and the license). See: https://creativecommons.org/licenses/by-nc-nd/4.0/.

\section{References}

1. Govindan R, Page N, Morgensztern D, et al. Changing epidemiology of small-cell lung cancer in the United States over the last 30 years: analysis of the surveillance, epidemiologic, and end results database. J Clin Oncol 2006;24:4539-44.

2. Jett JR, Schild SE, Kesler KA, et al. Treatment of small cell lung cancer: Diagnosis and management of lung cancer, 3rd ed: American College of Chest Physicians evidence-based clinical practice guidelines. Chest 2013;143:e400S-19S.

3. Pelayo Alvarez M, Westeel V, Cortes-Jofre M, et al. Chemotherapy versus best supportive care for extensive small cell lung cancer. Cochrane Database Syst Rev 2013;(11):CD001990.

4. Chung HC, Piha-Paul SA, Lopez-Martin J, et al. Pembrolizumab After Two or More Lines of Previous Therapy in Patients With Recurrent or Metastatic SmallCell Lung Cancer: Results From the KEYNOTE-028 and KEYNOTE-158 Studies. J Thorac Oncol 2020;15:618-27.

5. Ready N, Farago AF, de Braud F, et al. Third-Line Nivolumab Monotherapy in Recurrent SCLC: CheckMate 032. J Thorac Oncol 2019;14:237-44.

6. Lin B, Song X, Yang D, et al. Anlotinib inhibits angiogenesis via suppressing the activation of VEGFR2, PDGFRbeta and FGFR1. Gene 2018;654:77-86.

7. Mantovani A, Allavena P, Sica A, et al. Cancer-related inflammation. Nature 2008;454:436-44.

8. McMillan DC. Systemic inflammation, nutritional status and survival in patients with cancer. Curr Opin Clin Nutr Metab Care 2009;12:223-6.

9. Deans C, Wigmore SJ. Systemic inflammation, cachexia and prognosis in patients with cancer. Curr Opin Clin Nutr Metab Care 2005;8:265-9.

10. Alifano M, Mansuet-Lupo A, Lococo F, et al. Systemic inflammation, nutritional status and tumor immune microenvironment determine outcome of resected nonsmall cell lung cancer. PLoS One 2014;9:e106914.

11. Shoji F, Takeoka H, Kozuma Y, et al. Pretreatment prognostic nutritional index as a novel biomarker in non-small cell lung cancer patients treated with immune 
checkpoint inhibitors. Lung Cancer 2019;136:45-51.

12. Onodera T, Goseki N, Kosaki G. [Prognostic nutritional index in gastrointestinal surgery of malnourished cancer patients]. Nihon Geka Gakkai Zasshi 1984;85:1001-5.

13. Nozoe T, Ninomiya M, Maeda T, et al. Prognostic nutritional index: a tool to predict the biological aggressiveness of gastric carcinoma. Surg Today 2010;40:440-3.

14. Jiang N, Deng JY, Ding XW, et al. Prognostic nutritional index predicts postoperative complications and longterm outcomes of gastric cancer. World J Gastroenterol 2014;20:10537-44.

15. Nozoe T, Kohno M, Iguchi T, et al. The prognostic nutritional index can be a prognostic indicator in colorectal carcinoma. Surg Today 2012;42:532-5.

16. Chan AW, Chan SL, Wong GL, et al. Prognostic Nutritional Index (PNI) Predicts Tumor Recurrence of Very Early/Early Stage Hepatocellular Carcinoma After Surgical Resection. Ann Surg Oncol 2015;22:4138-48.

17. Ozdemir Y, Topkan E, Mertsoylu H, et al. Low Prognostic Nutritional Index Predicts Poor Clinical Outcomes in Patients with Stage IIIB Non-small-cell Lung Carcinoma Undergoing Chemoradiotherapy. Cancer Manag Res 2020;12:1959-67.

18. Hong S, Zhou T, Fang W, et al. The prognostic nutritional index (PNI) predicts overall survival of small-cell lung cancer patients. Tumour Biol 2015;36:3389-97.

19. Zhou T, Zhao Y, Zhao S, et al. Comparison of the Prognostic Value of Systemic Inflammation Response Markers in Small Cell Lung Cancer Patients. J Cancer 2019;10:1685-92.

20. Jin S, Cao S, Xu S, et al. Clinical impact of pretreatment prognostic nutritional index (PNI) in small cell lung cancer patients treated with platinum-based chemotherapy. Clin Respir J 2018;12:2433-40.

21. Wang Z, Wang Y, Zhang X, et al. Pretreatment prognostic nutritional index as a prognostic factor in lung cancer: Review and meta-analysis. Clin Chim Acta 2018;486:303-10.

22. Nagai S, Mangus RS, Kubal CA, et al. Prognosis after recurrence of hepatocellular carcinoma in liver transplantation: predictors for successful treatment and survival. Clin Transplant 2015;29:1156-63.

23. Hatanaka T, Kakizaki S, Uehara D, et al. Impact of the Prognostic Nutritional Index on the Survival of Japanese Patients with Hepatocellular Carcinoma Treated with Sorafenib: A Multicenter Retrospective Study. Intern Med 2019;58:1835-44.

24. Cai W, Zhong H, Kong W, et al. Significance of preoperative prognostic nutrition index as prognostic predictors in patients with metastatic renal cell carcinoma with tyrosine kinase inhibitors as first-line target therapy. Int Urol Nephrol 2017;49:1955-63.

25. Hong X, Cui B, Wang M, et al. Systemic Immuneinflammation Index, Based on Platelet Counts and Neutrophil-Lymphocyte Ratio, Is Useful for Predicting Prognosis in Small Cell Lung Cancer. Tohoku J Exp Med 2015;236:297-304.

26. Landskron G, De la Fuente $M$, Thuwajit $P$, et al. Chronic inflammation and cytokines in the tumor microenvironment. J Immunol Res 2014;2014:149185.

27. Li G, Gao J, Liu ZG, et al. Influence of pretreatment ideal body weight percentile and albumin on prognosis of nasopharyngeal carcinoma: Long-term outcomes of 512 patients from a single institution. Head Neck 2014;36:660-6.

28. Dalia S, Chavez J, Little B, et al. Serum albumin retains independent prognostic significance in diffuse large B-cell lymphoma in the post-rituximab era. Ann Hematol 2014;93:1305-12.

29. Biswas B, Rastogi S, Khan SA, et al. Hypoalbuminaemia is an independent predictor of poor outcome in metastatic Ewing's sarcoma family of tumours: a single institutional experience of 150 cases treated with uniform chemotherapy protocol. Clin Oncol (R Coll Radiol) 2014;26:722-9.

30. Lin JX, Lin JP, Xie JW, et al. Complete blood count-based inflammatory score (CBCS) is a novel prognostic marker for gastric cancer patients after curative resection. BMC Cancer 2020;20:11.

31. Hoshimoto S, Hishinuma S, Shirakawa H, et al. Validation and clinical usefulness of pre- and postoperative systemic inflammatory parameters as prognostic markers in patients with potentially resectable pancreatic cancer. Pancreatology 2020;20:239-46.

32. Chang WC, Yang CY, Lin CS, et al. Pretreatment body mass index as a prognostic predictor in patients with oral squamous cell carcinoma. Clin Oral Investig 2020;24:2781-8.

33. Farolfi A, Petrone M, Scarpi E, et al. Inflammatory Indexes as Prognostic and Predictive Factors in Ovarian Cancer Treated with Chemotherapy Alone or Together with Bevacizumab. A Multicenter, Retrospective Analysis by the MITO Group (MITO 24). Target Oncol 2018;13:469-79.

Cite this article as: Liu J, Li S, Zhang S, Yang C, Zhang L, Zhang B, Cheng Y, Wang C. Pretreatment prognostic nutritional index is a prognostic marker for extensive-stage small cell lung cancer patients treated with anlotinib. J Thorac Dis 2020;12(10):5765-5773. doi: 10.21037/jtd-20-755 